\title{
Spherical Wave Based Macro-Models for Efficient System-Level EMC Analysis in Circuit Simulators Part I: Optimized Derivation and Truncation Criteria
}

\author{
Bart Boesman, Georges G.E. Gielen, Guy A.E. Vandenbosch, and Davy Pissoort
}

\begin{abstract}
This work presents a general framework that enables one to compute near- and far-field interactions inside a circuit simulator environment, aimed at efficient system-level EMC analysis. For readability, the paper is subdivided in two parts. Part I focuses on deriving (high-order) spherical wave based macro-models in optimal time and describes two truncation criteria which define the maximum complexity of the models. The latter is generally a compromise between the accuracy of reconstructed near-fields and the computation time for evaluating interactions between these models, and is therefore a crucial parameter when such models are to be applied in fast circuit solvers. Existing guidelines on truncating spherical wave expansions are held against the newly derived criteria. In part II, the framework is extended with optimized routines that enable one to compute efficiently near- and far-field interactions between the macro-models discussed in part I. It will be shown that by extensive understanding of spherical wave expansions, the underlying computations only add negligible computational cost compared to traditional S-parameter simulations, partially contributed to correctly chosen truncations of the applied models.
\end{abstract}

Index Terms-System-Level EMC Analysis, Macro-Modeling, Generalized Scattering Matrix, Spherical Wave Expansion, Truncation Criteria.

\section{INTRODUCTION}

Electromagnetic (EM) simulation tools offer electrical engineers and designers an increasing level of flexibility as well as an increasing number of analysis techniques applicable to their product design and validation towards Electromagnetic Compatibility (EMC) compared to traditional measurements in reverberant or (semi-)anechoic chambers. Where the latter only allow an engineer to mark a product as passed or failed, simulation techniques such as Finite Difference Time Domain (FDTD), Finite Element Method (FEM), and Method of Moments (MoM) allow one to visualize and predict unwanted phenomena which possibly lead to a failed result in a certified EMC test lab. However, simulating a full model of a real electrical design, which in general consists of small details (narrow traces and vias, integrated circuits, small active and passive components), electrically large (multi-layer) Printed Circuit Boards (PCBs) and long complex cable bundles, would demand too large an amount of computational resources. Therefore, existing commercial EM tools [1]-[5] try to overcome these problems by offering (often advanced) modules which enable a user to extract sub-parts and afterwards recombine separate results - both obtained from simulations as from custom measurements - using appropriate sub-level and system-level simulation engines. Examples of such 'hybrid' simulation techniques are among others equivalent dipole based engines [6]-[8]; source-reconstruction techniques based on equivalent current distributions [9] or combined circuit/fullwave simulation engines [10], [11].

Among these 'hybrid' techniques, the expansion wave concept is extensively used in literature to more efficiently model EM emission, immunity and coupling and is applied to a wide range of problems [12]-[15]. Spherical Wave Expansions [16] (SWEs) are of special interest in EMC analysis as one is commonly confronted with unwanted radiation from arbitrary Devices Under Test (DUTs) in contrast to e.g. antenna analysis, in which one often has prior knowledge about the radiation pattern of an antenna. Moreover, a SWE formulation is easily linked to a set of equivalent scattering parameters ( $\mathrm{S}$ parameters). Subsequently, S-parameters can be used to model or simulate not only voltages and currents at a DUT's circuit ports, but also fields radiated by the DUT, and this in a very straightforward manner. After all, the entire full-wave analysis can be hidden for the user, who can simply 'plug and play' with models available from a common library, as he is used to work with in currently existing circuit simulators.

The well-known theory of SWEs is used in the first part of two papers to create a library of S-parameter based macromodels which take into account (unwanted) emission from a DUT. Contrary to current applications of SWE-based models, the novelty of the presented work lies in (i) a new workflow that combines the derivation of high-order models in optimal time and two truncation criteria; and (ii) applying these macromodels in fast EM circuit solvers taking into account emission and mutual coupling effects between models without affecting computation times compared to traditional S-parameter simulations. It is shown that by optimally truncating the models, their complexity (i.e. number of modes) is minimized without compromising accuracy of reconstructed fields or the evaluation of model interactions. In SWE theory, it is generally suggested to truncate a model at a SWE order $N=k R_{0}+n_{1}$ [17]-[19], where $k$ is the wave number, $R_{0}$ the radius of the minimum-sphere enclosing all sources and $n_{1}$ a constant commonly set to 10 . Two references are found in literature [17], [20] that cite an estimate of $n_{1}$ as $O\left(\sqrt[3]{k R_{0}}\right)$, but no validation is given. Nevertheless, this paper will show that due to the broadband and unintentional radiation characteristics of DUTs in EMC problems, this truncation criterion will not suffice and subsequently two new truncation criteria are derived: one based on the power radiated by a DUT in its far-field region and a second one based on the stored reactive 


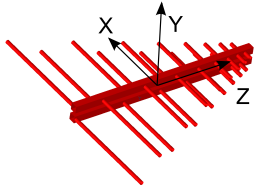

(a)

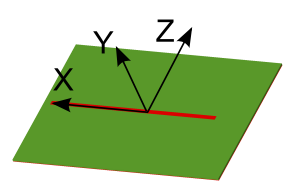

(b)

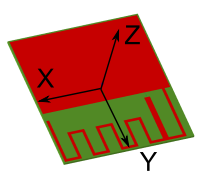

(c)

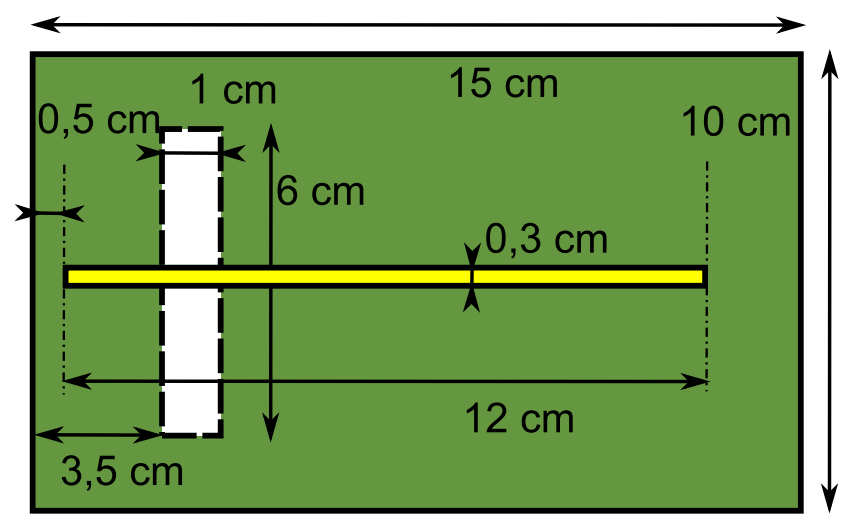

(d)

Fig. 1. The log-periodic antenna (a), the PCB (b) and the inverted-F antenna (c) in their respective coordinate systems, modeled using Keysight Empro [23]. A close-up of the PCB dimensions is shown in (d).

energy present in the near-field region of a DUT.

Based on the work presented in this paper, the second part [21] will apply SWE based macro-models in fast EM circuit solvers and discuss (i) computing general interactions in optimal time based on optimized routines and a thorough understanding of SWE theory; and (ii) adapting the presented routines to evaluate a full cylindrical measurement set-up of a DUT with negligible increase in computation time compared to (i).

Part I starts with a recapitulation of SWE based macromodels in section II, followed by a discussion on the energy flow around a DUT in Section III. Based on expressions for the radiated power and the stored reactive energy in function of a SWE, truncation criteria for the presented SWE based macro-models are proposed in Section IV. These criteria are validated by reconstructing the near-field on a minimumsphere enclosing the DUT being modeled from its SWE. Three examples are studied (see Figure 1): (i) a log-periodic antenna that is commonly used in EMC measurements (Type: ETS Lindgren EMCO 3146); (ii) a PCB comprising a trace routed over a gap; and (iii) an on-board inverted-F antenna e.g. used in cellphones. A detailed description of the Inverted-F antenna can be found in [22]. For the latter, a substrate thickness of 0.8 $\mathrm{mm}$ has been selected, while the PCB thickness amounts to $1.6 \mathrm{~mm}$. All examples are analyzed between $200 \mathrm{MHz}$ and 2 GHz. In what follows, matrices are denoted by bold symbols.

\section{SWE BASED MACRO-MOdel OF A DUT}

\section{A. Background}

Due to the discrete character of a SWE, it perfectly fits to the widespread known S-parameter format commonly used for
Radio Frequency (RF) analysis. This implies the possibility to define 'radiation ports' able to transmit and receive spherical waves, rendering a definition of a Generalized Scattering Matrix [17]-[19], [24]:

$$
\mathbf{S}_{d u t}=\left(\begin{array}{cc}
\mathbf{S}_{e l}[U \times U] & \mathbf{S}_{i n c}[U \times V] \\
\mathbf{S}_{\text {rad }}[V \times U] & \mathbf{S}_{\text {scat }}[V \times V]
\end{array}\right)
$$

This Generalized Scattering Matrix (GS-Matrix) consists of an electrical S-matrix $\mathbf{S}_{e l}$, a radiation S-matrix $\mathbf{S}_{\text {rad }}$, an incidence S-matrix $\mathbf{S}_{i n c}$ and a scattering S-matrix $\mathbf{S}_{\text {scat }}$. A DUT's SWE based macro-model is built by computing a set of 'radiation' S-parameters that link the incident waves $a_{j}$ at the DUT's $U$ circuit ports to $U$ collections of $V$ radiated spherical waves $Q_{i, j}$ :

$$
S_{r a d, i, j}=\frac{Q_{i, j}}{a_{j}}
$$

As such, a $(V \times U)$ matrix is created, where $U$ is the number of circuit ports and $V$ the number of radiation ports (or spherical waves). All spherical waves are computed from sampled near-field data on the minimum-sphere of the DUT, which encloses all radiating sources or sampled far-field data at infinity, using an optimized routine described in Section II-B. How both sets of spherical waves are related to each other will be discussed in Section III. Due to reciprocity [17], $\mathbf{S}_{i n c}$, which relates incident spherical waves to outgoing waves $b_{j}$ at the $U$ circuit ports, can be derived directly from $\mathbf{S}_{r a d}$. Furthermore, $\mathbf{S}_{\text {scat }}$, representing scattering of incident spherical waves, can be assumed to equal the zero-matrix. After all, the low directionality of emitted energy in common EMC problems will ensure that the twice back-scattered energy is low enough. As a result, the GS-Matrix can be derived from one full-wave simulation when the DUT is transmitting.

\section{B. Optimized Derivation}

Among the different formulations and definitions for SWEs being used in literature, the definition and power-normalization according to [17] is used in the work below (see Appendix A). Although the most common approach to compute a SWE is by point matching of (spherically) sampled field data and matrix inversion, it is shown in [25] that this method fails when the number of spherical waves becomes large or when the radius of the spherically sampled data becomes very small. A more efficient and numerically stable approach is to make use of the orthogonality properties of spherical wave functions and apply a smart combination of a Fast Fourier Transform (FFT), a Discrete Cosine Transform (DCT) and a Discrete Sine Transform (DST) of the captured fields, and this in contrast to more conventional quadrature routines.

TABLE I

COMPUTATION TIME SWE BASED MACRO-MODELS (IN SECONDS)

\begin{tabular}{c|cccc}
\hline & $\mathbf{N = 2 5}$ & $\mathbf{N = 5 0}$ & $\mathbf{N = 7 5}$ & $\mathbf{N = 1 0 0}$ \\
\hline \hline SWE Electric Near-field & 1.5 & 5.2 & 11.6 & 21.4 \\
SWE Magnetic Near-field & 1.5 & 5.5 & 12.1 & 22.3 \\
SWE Electric Far-field & 1.2 & 3.6 & 10.2 & 17.8
\end{tabular}




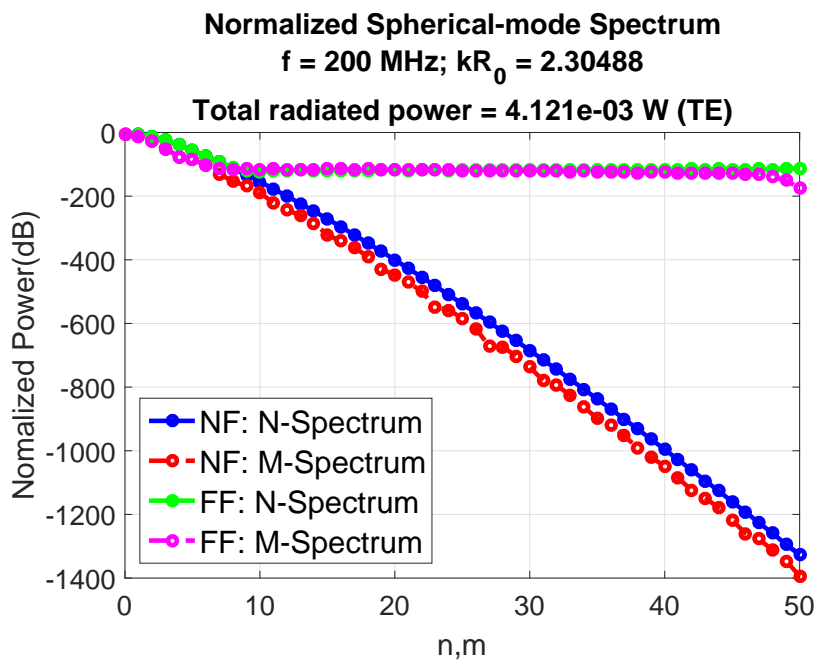

(a)

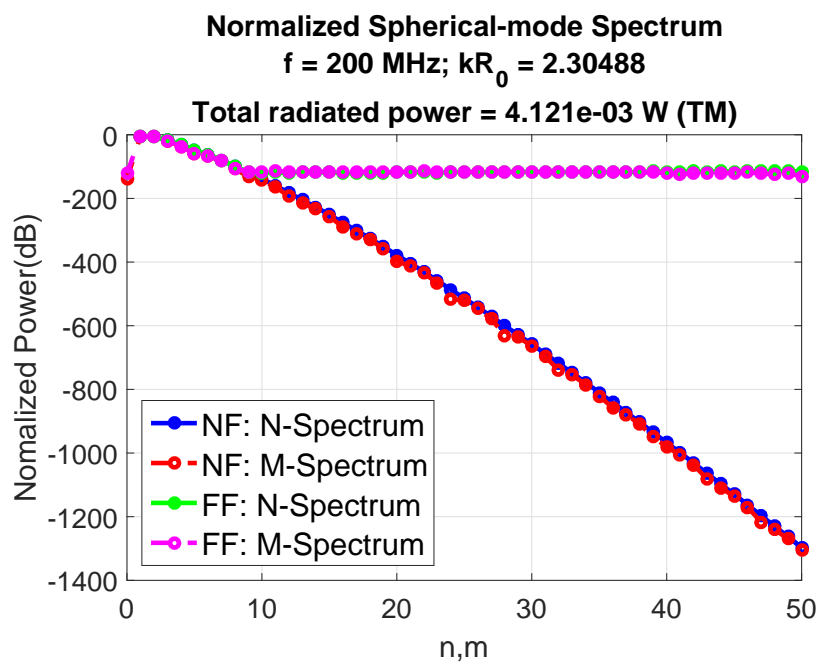

(b)

Fig. 2. The TE(a) and TM(b) N- and M- radiated power spectra of the logperiodic antenna obtained from both near-field (NF) data and far-field (FF) data.

1) Expanding near-field data: Based on the expressions given in Appendix A and the orthogonality properties of spherical wave functions [17], the general derivation of the SWE from a set of tangential electrical near-field data $\left(E_{\phi}, E_{\theta}\right)$ captured on a sphere with radius $r$ is computed as:

$$
\begin{aligned}
& Q_{s=1, m, n}^{(3)}=\frac{\sqrt{\eta}}{k} \frac{(-1)^{m}}{\sqrt{2 \pi n(n+1)}} \frac{1}{R_{1 n}^{(3)}(k r)}\left(\frac{m}{|m|}\right)^{-m} \times \\
& \left\{-(i \cdot m) I_{1}\left(E_{\theta}(\theta, \phi)\right)-I_{2}\left(E_{\phi}(\theta, \phi)\right)\right\} \\
& Q_{s=2, m, n}^{(3)}=\frac{\sqrt{\eta}}{k} \frac{(-1)^{m}}{\sqrt{2 \pi n(n+1)}} \frac{1}{R_{2 n}^{(3)}(k r)}\left(\frac{m}{|m|}\right)^{-m} \times \\
& \left\{-(i \cdot m) I_{1}\left(E_{\phi}(\theta, \phi)\right)+I_{2}\left(E_{\theta}(\theta, \phi)\right)\right\}
\end{aligned}
$$

In above expressions, $Q_{s=1, m, n}^{(3)}$ and $Q_{s=2, m, n}^{(3)}$ represent outward propagating spherical waves - distinguishing between a Transverse Electric (TE) mode $(\mathrm{s}=1)$ and a Transverse Magnetic (TM) mode (s=2) -, $k$ is the wave number, $\eta$ is the free-space admittance, and $n$ and $m$ respectively denote the order and degree of a spherical wave. Further, the integrals $I_{1}(f(\theta, \phi))$ and $I_{2}(f(\theta, \phi))$ represent twice-transformed field data on a spherical grid with radius $r$. To optimally evaluate $I_{1}$ and $I_{2}$, first the one-dimensional FFT $\tilde{f}(\theta, m)$ of the data $f(\theta, \phi)$ in $\phi$ is taken, which is subsequently transformed into its Discrete Sine (DST) or Discrete Cosine (DCT) transform, depending on the parity of the mode's degree $m$ being computed:

$$
\begin{aligned}
& I_{1}=\int_{0}^{\pi} \tilde{f}(\theta, m) \frac{m \bar{P}_{n}^{|m|}(\cos \theta)}{\sin \theta} \sin \theta d \theta=\int_{-1}^{1} \sum_{k=0}^{k_{\max }} y_{k} T_{k}(x) d x \\
& I_{2}=\int_{0}^{\pi} \tilde{f}(\theta, m) \frac{d \bar{P}_{n}^{|m|}(\cos \theta)}{d \theta} \sin \theta d \theta=\int_{-1}^{1} \sum_{k=0}^{k_{\max }^{\prime}} y_{k}^{\prime} T_{k}(x) d x
\end{aligned}
$$

For $m$ being even, $\tilde{f}(\theta, m)$, as well as $\frac{m \bar{P}_{n}^{|m|}(\cos \theta)}{\sin \theta}$ and $\frac{d \bar{P}_{n}^{|m|}(\cos \theta)}{d \theta}$ are being transformed to sine series, while for odd $m$, cosine series are used. In other words, the integrands in $I_{1}$ and $I_{2}$ reduce to series of Chebyshev polynomials $T_{k}(x)$, allowing one to reduce the integrals $I_{1}(f(\theta, \phi))$ and $I_{2}(f(\theta, \phi))$ to simple sums over respectively Chebyshev coefficients $y_{k}$ and $y_{k}^{\prime}$. As a consequence, near-field data on a spherical grid is efficiently but exactly expanded into a set of spherical waves in the order of seconds. Due to the sampling criteria of the transforms, minimal $2 N+1$ samples in $\theta$ and $\phi$ are necessary, $N$ being the bandwidth of the model. Remark that $N$ should be taken sufficiently high in order to avoid under-sampling. More details on choosing $N$ will be given in Section IV. Truncating at $N=50$ - equivalent to $2 N(N+2)$ or 5200 modes - using 301 samples in both $\theta$ and $\phi$ takes about 5.2 seconds on a dual core $2.1 \mathrm{GHz}$ processor, as shown in Table I. From this table, a linear relationship can be found between the number of unknowns $(=2 N(N+2))$ and the computation time of a model using the approach described above.

In equations (4a) and (4b), it is assumed that the sine and cosine transforms of Associated Legendre Functions (ALFs) are available before computation. To avoid transforming a large set of ALFs each time, new relations are derived in Appendix B that recursively compute the DST or DCT of ALFs, depending on the parity of degree $m$. Multiplying two sine or cosine series is optimally implemented as the convolution of two fourier series.

Equations (3a) and (3b) can easily be adapted to expand sampled magnetic fields on the minimum-sphere of the DUT using the relation between $\vec{E}$ and $\vec{H}$ in Equation (12).

2) Expanding far-field data: Instead of near-field data on the minimum-sphere, field data on a sphere with any radius can be used to evaluate equations (3a) and (3b). Therefore, also the far-field pattern can be expanded into a set of spherical waves: 


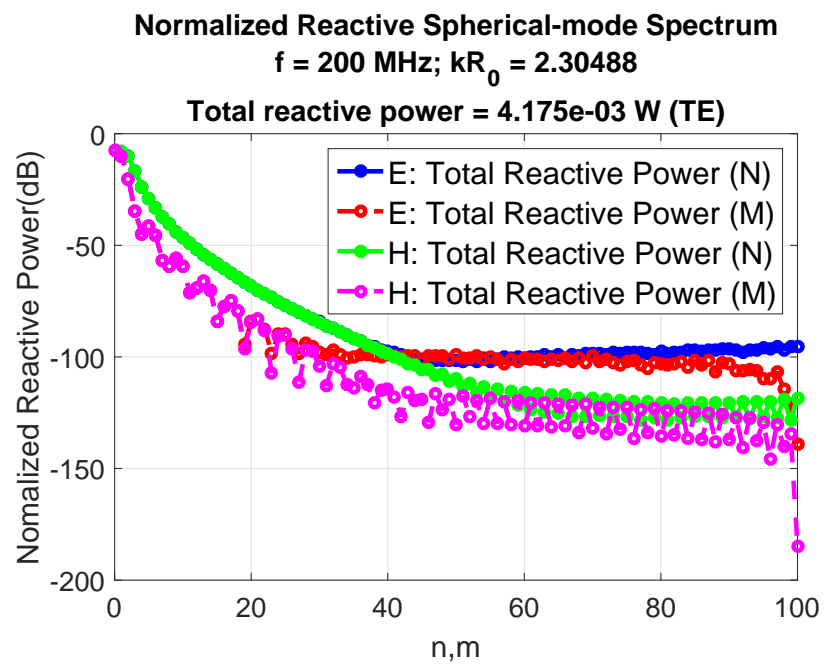

(a)

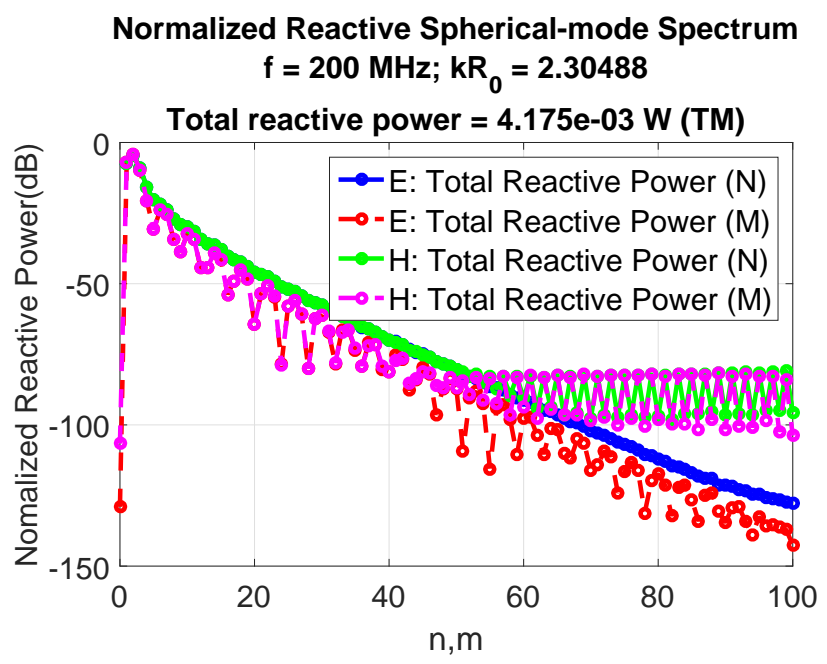

(b)

Fig. 3. TE(a) and TM(b) N- and M- reactive power spectra of the log-periodic antenna obtained from electric $(\mathrm{E})$ and magnetic $(\mathrm{H})$ near-field data.

$$
\begin{aligned}
& Q_{s=1, m, n}^{(3)}=\sqrt{\frac{\eta}{4 \pi}}(-1)^{m} \sqrt{\frac{2}{n(n+1)}} \frac{1}{(-i)^{n+1}} \\
& \left(\frac{m}{|m|}\right)^{-m}\left\{-(i \cdot m) I_{1}\left(E_{\theta}(\theta, \phi)\right)-I_{2}\left(E_{\phi}(\theta, \phi)\right)\right\} \\
& Q_{s=2, m, n}^{(3)}=\sqrt{\frac{\eta}{4 \pi}}(-1)^{m} \sqrt{\frac{2}{n(n+1)}} \frac{1}{(-i)^{n}} \\
& \left(\frac{m}{|m|}\right)^{-m}\left\{-(i \cdot m) I_{1}\left(E_{\phi}(\theta, \phi)\right)+I_{2}\left(E_{\theta}(\theta, \phi)\right)\right\}
\end{aligned}
$$

In equation (5), $E_{\theta}$ and $E_{\phi}$ represent the sampled far-field pattern of a DUT. Evidently, the obtained expansion will form a subset of the expansion derived using near-field data of the DUT. This will be explored more thoroughly in the following section.

\section{Electromagnetic ENERGies IN THE NEAR-FIELD AND FAR-FIELD REGION}

The power flow in time-harmonic fields is described by the complex Poynting vector:

$$
\vec{S}=\frac{1}{2} \vec{E} \times \vec{H}^{*}
$$

By substituting the SWE of electric and magnetic fields and integrating equation (6) over a spherical surface with radius $r$, one obtains a complex quantity associated with the complex power flow through a surface $S$ :

$$
\begin{aligned}
& \int_{0}^{2 \pi} \int_{0}^{\pi} \vec{S} \cdot \hat{r} r^{2} \sin \theta d \theta d \phi \\
= & \sum_{s m n}\left\{\frac{1}{2}+\frac{1}{2} i(-1)^{3-s}(k r)^{2}\left(\frac{1}{k r}+\frac{d}{d(k r)}\right) .\right. \\
& \left.\left|h_{n}^{(1)}(k r)\right|^{2}\right\}\left|Q_{s m n}^{(3)}\right|^{2} \\
= & P_{r a d}+2 i \omega\left(W_{e}-W_{m}\right)
\end{aligned}
$$

Equation (7) relates the radiated power and the net timeaverage stored reactive energy in the field of a DUT to its expansion coefficients. Given that the radiated power and the net stored reactive energy are real quantities, and observing that the quantity depending on $k r$ in Equation (7) can be shown to be always finite [17], it follows that a DUT's SWE is always bandwidth-limited. In the following two subsections, the bandwidth-limitation of an arbitrary DUT's SWE, both computed from near-field and far-field data, will be verified using a simplified wire-model of the log-periodic antenna, modeled in NEC [26]. In addition to the net reactive stored energy, expressions for the total reactive stored energy are given in section III-B. In section IV, the results in this section will be used to present a set of new approaches to correctly truncate the expansion aiming to reconstruct the near-field on the minimum-sphere of a DUT.

\section{A. Radiated Power}

Based on the expression of the radiated power in Equation (7) one is able to define the normalized radiated power $\mathrm{N}$ spectrum and M-spectrum as:

$$
\begin{aligned}
P_{n\{T E, T M\}}^{n o r m} & =\frac{\sum_{m=-n}^{n}\left|Q_{s=\{1,2\} m n}^{(3)}\right|^{2}}{P_{\text {rad }}} \\
P_{m\{T E, T M\}}^{n o r m} & =\frac{\sum_{n=|m|}^{N}\left|Q_{s=\{1,2\} m n}^{(3)}\right|^{2}}{P_{\text {rad }}}
\end{aligned}
$$

Both spectra are normalized to the total radiated power Prad.

Figure 2 shows the computed TE and TM radiated power spectra of the log-periodic antenna at $200 \mathrm{MHz}$, computed both from near-field and far-field data. One remarks that the total radiated power converges to a constant value when taking into account an increasing number of modes. After all, the power in the highest orders is sufficiently low compared to the power in the lower orders. The monotonically decreasing behavior of the near-field spectra for large values of a mode's 


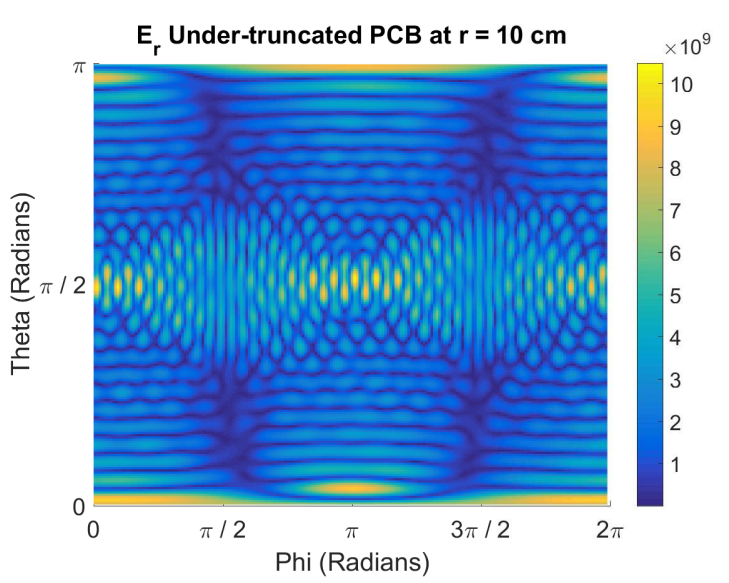

(a)

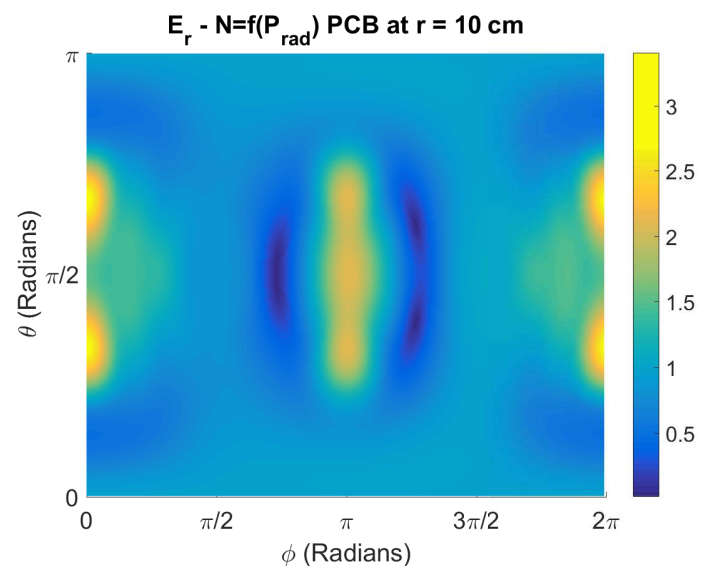

(c)

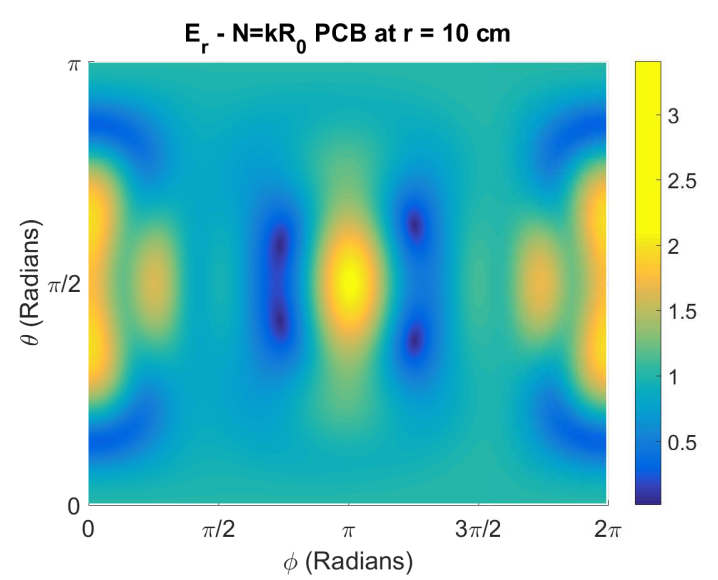

(b)

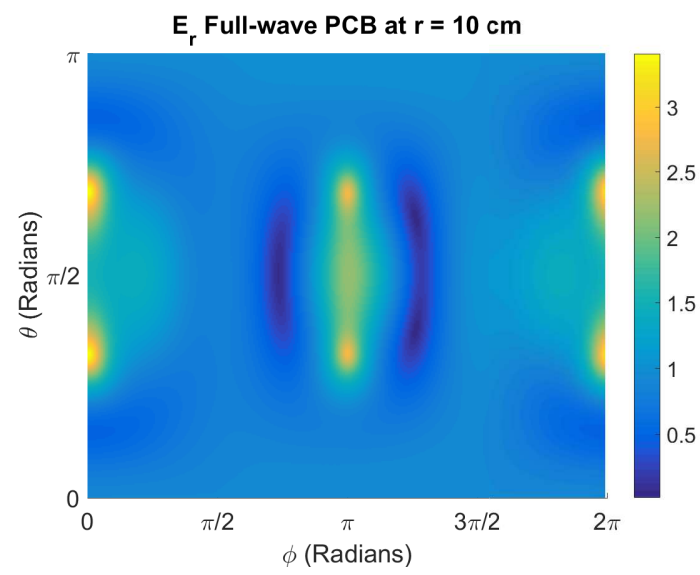

(d)

Fig. 4. Reconstructed near-field $\left(E_{r}\right)$ in V/m on the minimum-sphere of the PCB, working at 2 GHz, computed from far-field data after under-truncating the model (a); after truncating the model at $N=\left\lceil k R_{0}\right\rceil$ (b); and after truncating the model based on the radiated power spectrum (c). In addition, the fields (in $\mathrm{V} / \mathrm{m}$ ) obtained using a full-wave solver are shown in $(\mathrm{d})$.

order $n$ and degree $m$ is caused by the large-valued Hankel functions in Equation (13) when $n$ and $m$ become much larger than $k r$.

The lower bound on the far-field spectra is due to the absence of modes higher than a certain truncation $n=N_{F F}$ - where $N_{F F}$ is a model-dependent constant extracted from a model's far-field spectra - in the radiated fields, resulting in numerical noise for $n>N_{F F}$. This is in accordance to the observation that only part of the energy being present in the near-field region of the DUT will effectively radiate. As a result, one possesses a starting point to truncate a computed spherical wave expansion. From theory, it is known that modes with $n \gg k R_{0}, R_{0}$ being the radius of the minimumsphere enclosing the DUT, are heavily attenuated outside this minimum radius. Figure 2 shows that an additional number of modes $n=k R_{0}+n_{1}$ is obtained from the available farfield data. In section IV, some examples will be given showing that in certain cases, the near-field can be reconstructed with extremely high precision from far-field data only. Moreover, it will be shown in [21] that it is possible to validate the computation of near-field interactions using models derived from far-field data.

\section{B. Reactive Energy}

By taking the imaginary part of the complex Poynting vector, one obtains the net time-average reactive energy flux through an elementary surface $d S$, being equal to the difference between the stored electric and magnetic energy flux. In the far-field region, the complex Poynting vector is purely real, implying the net reactive energy to equal zero. Spherical modes with a degree $n \leq k r$ represent propagating modes, having therefore a net reactive energy close to zero. However modes with $n \gg k r$ are evanescent, meaning that a TE-mode will have its stored magnetic energy to be larger than its stored electric energy and inversely for a TM-mode. Subsequently, these modes will have a net reactive energy about equal to the larger quantity of its electric and magnetic stored energy. Due to these properties, the spherical wave spectrum of the net stored reactive energy shows a rather flattened trend reaching from $n=0$ towards $\infty$. Because in this paper a truncation criterion is of main interest, the further discussion focuses on the total stored reactive energy which is present outside the minimum radius of the DUT. This quantity will prove to be a good measure for the bandwidth of a near-field dataset. The sum of the electric and magnetic energy density in the region 
outside the minimum-sphere of a DUT is obtained by solving the integral below:

$$
\begin{aligned}
& W_{t o t}=\int_{r_{0}}^{\infty} \int_{0}^{2 \pi} \int_{0}^{\pi}\left\{\frac{\epsilon_{0}}{4}|\vec{E}|^{2}+\frac{\mu_{0}}{4}|\vec{H}|^{2}\right\} r^{2} \\
& \sin \theta d \theta d \phi d r
\end{aligned}
$$

As the integrand in Equation (9) also includes the radiated energy, the integrand converges to a non-zero value for $k r \rightarrow$ $\infty$, leading to an undefined integral. This can nevertheless be avoided by subtracting the electric and magnetic energy in the far-field using the expressions in Equation (17). Finally one is left over with the sum of the stored reactive electric and magnetic energy in the fields:

$$
\begin{aligned}
& W_{\text {reactive }}=\frac{1}{4 \omega} \sum_{s m n}\left|Q_{s m n}^{(3)}\right|^{2} \int_{k R_{0}}^{\infty}\left\{\left[R_{1 n}^{(3)}(k r) R_{1 n}^{(4)}(k r)+\right.\right. \\
& \left.R_{2 n}^{(3)}(k r) R_{2 n}^{(4)}(k r)\right](k r)^{2}-2+n(n+1) . \\
& \left.z_{n}^{(3)}(k r) z_{n}^{(4)}(k r)\right\} d(k r)
\end{aligned}
$$

Numerical quadrature rules can be used to solve the integral in Equation (10), because the integral asymptotically approaches zero for $k r \rightarrow \infty$. In literature, only a few references [27], [28] provide analytical expressions for this integral, although no direct derivation is given. Main disadvantage of using numerical quadrature or analytical expressions to evaluate (10) is that the products of Hankel functions lead to numerical overflow for large $n$ and small $k r$. A workaround is to write (10) as a finite series $\sum_{i=1, i=o d d}^{2 n+1} c_{i} /(k r)^{i}$, and to combine series truncation, scaling and conversion to a logarithmic scale in order to avoid numerical overflow.

Multiplying $W_{\text {reactive }}$ by the angular frequency $\omega$ gives the total reactive power and will be used further as the main quantity characterizing the near-field of a DUT. The reactive power spectra $\omega W_{n\{T E, T M\}}^{n o r m}\left(\mathrm{~N}\right.$-spectrum) and $\omega W_{m\{T E, T M\}}^{n o r m}(\mathrm{M}-$ spectrum) are defined analogous to the radiated power spectra but normalized to the total reactive power $\omega W_{\text {reactive }}$ :

$$
\begin{aligned}
& \omega W_{n\{T E, T M\}}^{\text {norm }}=\frac{1}{4 \omega W_{\text {reactive }}} \sum_{m=-n}^{n}\left|Q_{s=\{1,2\} m n}^{(3)}\right|^{2} \\
& \int_{k R_{0}}^{\infty}\left\{\left[R_{1 n}^{(3)}(k r) R_{1 n}^{(4)}(k r)+R_{2 n}^{(3)}(k r) R_{2 n}^{(4)}(k r)\right](k r)^{2}\right. \\
& \left.+n(n+1) z_{n}^{(3)}(k r) z_{n}^{(4)}(k r)-2\right\} d(k r) \\
& \omega W_{m\{T E, T M\}}^{n o r m}=\frac{1}{4 \omega W_{\text {reactive }}} \sum_{n=|m|}^{N}\left|Q_{s=\{1,2\} m n}^{(3)}\right|^{2} \\
& \int_{k R_{0}}^{\infty}\left\{\left[R_{1 n}^{(3)}(k r) R_{1 n}^{(4)}(k r)+R_{2 n}^{(3)}(k r) R_{2 n}^{(4)}(k r)\right](k r)^{2}\right. \\
& \left.+n(n+1) z_{n}^{(3)}(k r) z_{n}^{(4)}(k r)-2\right\} d(k r)
\end{aligned}
$$

Note that $W_{\text {reactive }}$ is also proportional to the quality factor by a factor $\omega / P_{\text {rad }}$, commonly used in antenna analysis as a measure for the performance of an antenna. Nevertheless, the discussion below will always use the reactive power spectrum which is normalized to the total reactive power, and not the quality factor spectrum in which the normalization factor

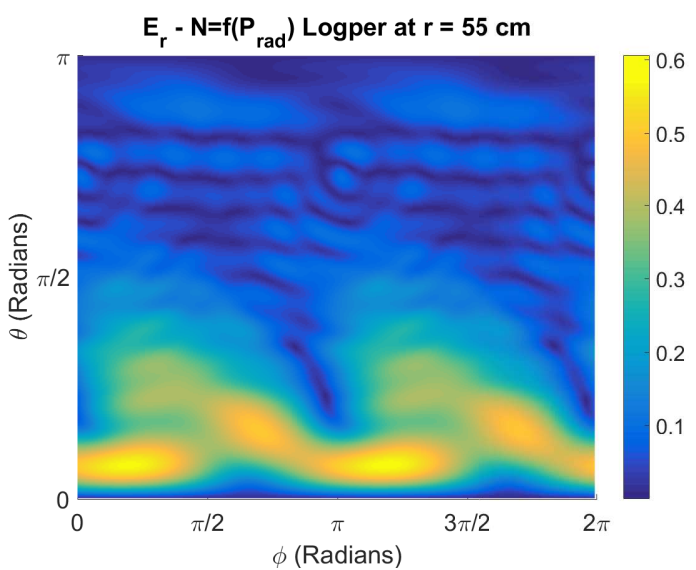

(a)

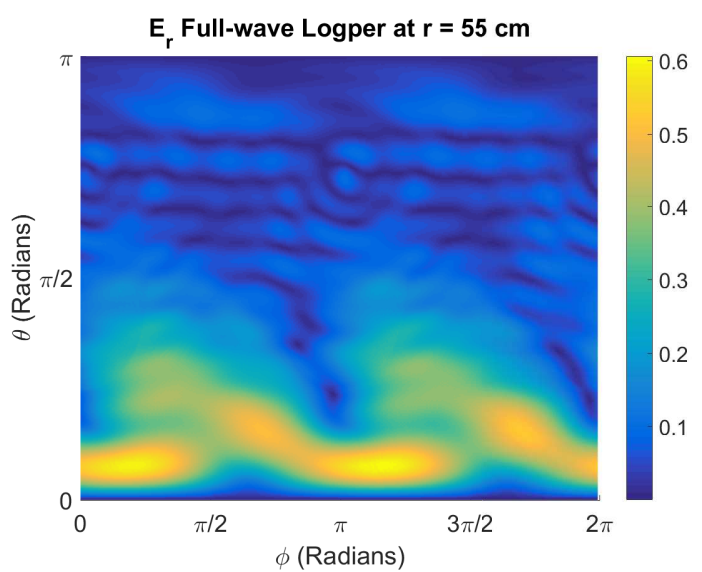

(b)

Fig. 5. Reconstructed near-field $\left(E_{r}\right)$ in V/m on the minimum-sphere of the log-periodic antenna, working at $2 \mathrm{GHz}$, computed from far-field data after truncating the model based on the radiated power spectrum (a), followed by the fields (in V/m) obtained using a full-wave solver (b).

equals the total radiated power. This choice implies that any truncation criterion based on these spectra will use the total reactive power as reference, which intuitively has a more clear link with the electric and magnetic field distribution in a DUT's near-field, as seen from equation (9), than the quality factor. Each value in the reactive power spectrum thus denotes the contribution of an order $n$ or degree $m$ to the field distribution in a DUT's near-field region. Observe from Figure 3 that, analogous to the radiated power spectrum of far-field data, the reactive power spectrum of near-field data asymptotically approaches a lower bound for increasing $n$. The latter observation suggests that the lower bound on the near-field spectra, analogous to the case of far-field radiated power spectra, is due to the high attenuation of modes higher than a certain truncation $n=N_{N F}$. More importantly, from a certain $n$ on, expanding E-fields and H-fields results in different spectra (see Figure 3). Section IV will verify that the correct TE-spectrum is only obtained from $\mathrm{H}$-fields, while the correct TM-spectrum has to be computed from E-fields. 


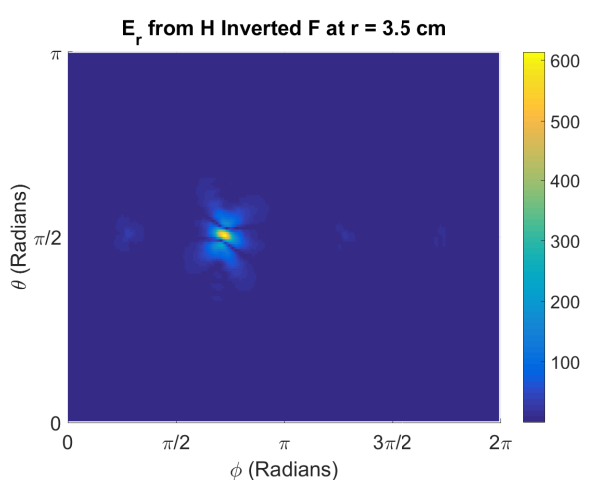

(a)

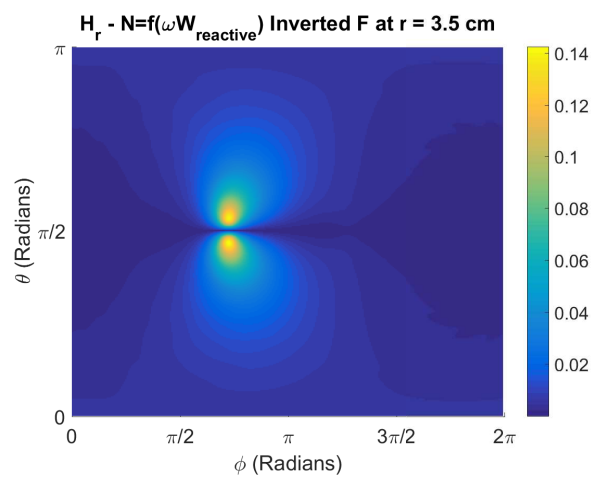

(c)

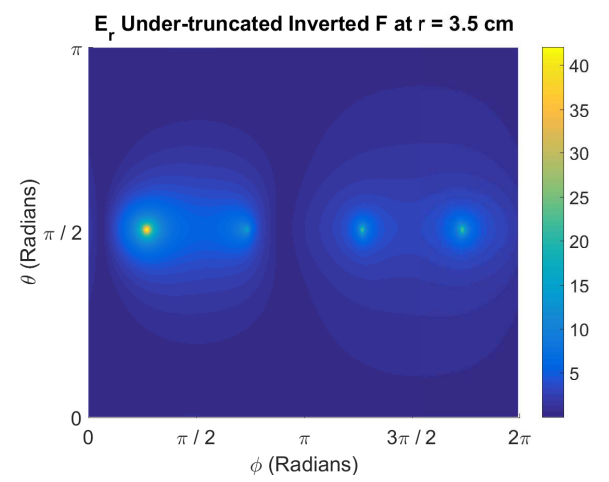

(e)

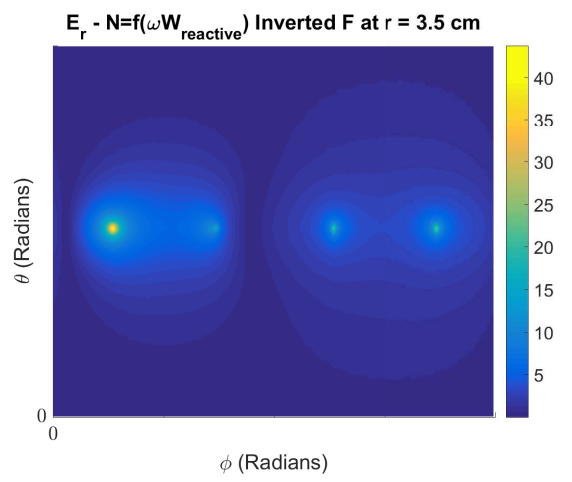

(g)

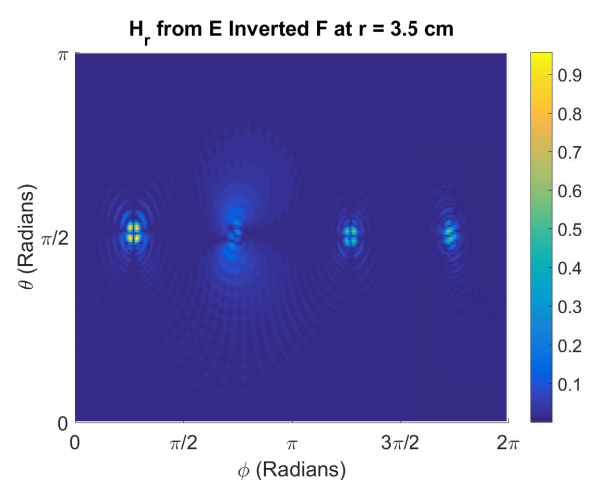

(b)

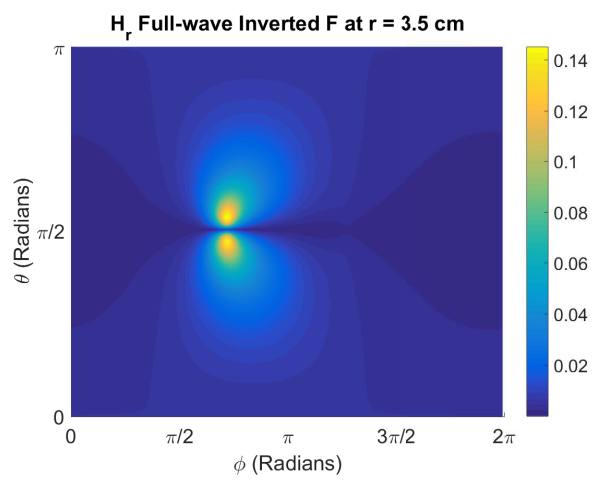

(d)

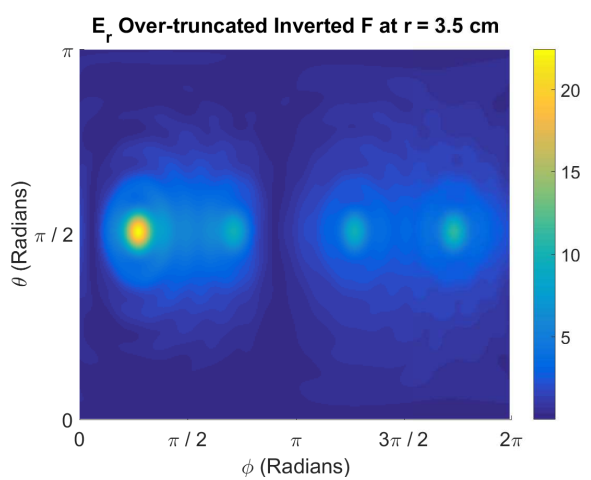

(f)

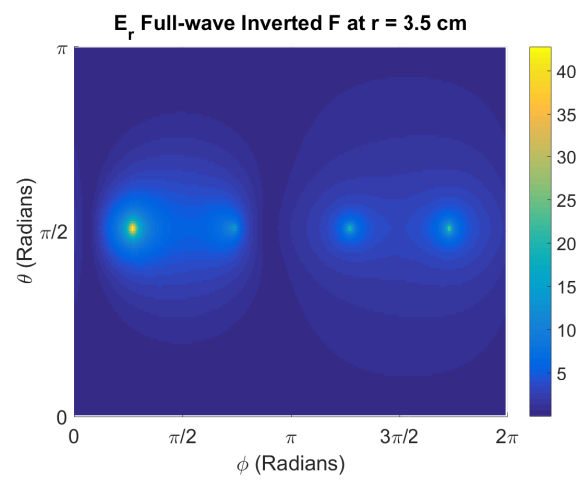

(h)

Fig. 6. Reconstructed electric $\left(E_{r}\right)$ (a) and magnetic $\left(H_{r}\right)$ (b) near-field on the minimum-sphere of the inverted-F antenna, working at $200 \mathrm{MHz}$, computed from magnetic and electric near-field data, respectively; reconstructed near-field $\left(E_{r}\right)$ on the minimum-sphere of the Inverted-F antenna, working at $200 \mathrm{MHz}$, computed using an under-truncated (e) and an over-truncated (f) model, both derived from both electric and magnetic near-field data; reconstructed near-field $\left(H_{r}\right.$ in $(\mathrm{c}), E_{r}$ in $\left.(\mathrm{g})\right)$ on the minimum-sphere of the Inverted-F antenna, working at $200 \mathrm{MHz}$ by truncating the model, derived from both electric and magnetic near-field data, based on the reactive power spectrum. For comparison, the magnetic and electric near-fields obtained using a full-wave solver are shown in (d) and (h), respectively. The electric fields are displayed in $\mathrm{V} / \mathrm{m}$ and the magnetic fields in $\mathrm{A} / \mathrm{m}$. 


\section{TRUnCATING SWE BASED MODELS}

This section describes a set of approaches to truncate models obtained by expanding near- and far-field data into spherical waves. Truncation is always split up into two separate steps: (i) TE-truncation and (ii) TM-truncation, in order to obtain a minimum number of modes. Equation (3) showed that the radial electric field component is constituted of TM-modes only while the radial magnetic component is constituted of TEmodes only. Due to the latter observation and for conciseness of the presented work, only the radial field components are shown in what follows, although all field components have been verified. Validation is done using the three DUTs shown in Figure 1 and modeled in the full-wave simulation tool Keysight Empro [23], with minimum-spheres having radii of $55 \mathrm{~cm}, 10 \mathrm{~cm}$ and $3.5 \mathrm{~cm}$ for the log-periodic antenna, the $\mathrm{PCB}$, and the inverted-F antenna, respectively.

\section{A. Far-field data}

In case of expanding far-field data, under-truncating the obtained model, leads to infinitely large errors due to the large amplification of erroneous high-order modes (see Section III-A). Therefore, if e.g. only far-field data are available to build a model, one needs a reliable method to truncate the computed expansion. Truncating at $M_{\{T E, T M\}}=\left\lceil k R_{0}\right\rceil$ will ensure a converged expansion, although Section III-A showed the presence of an additional set modes in the radiated power spectra. Therefore, the latter corresponds to over-truncating far-field data.

In order to remove the erroneous tail of the computed expansion, only the modes for which $P_{m\{T E, T M\}}^{n o r m}(\mathrm{~dB})>A_{F F}(\mathrm{~dB})$ are kept. In this notation, $F F$ refers to expanding far-field data, while $A$ denotes a constant threshold which is applicable only on the $M$-spectrum of a model. Reason for choosing the M-spectrum is the sequence in computing the expansion, outlined in section II-B. Thereupon, it becomes possible to truncate $N$ independently from $M$. After all, setting $N$ equal to $M$ would mean that theoretically the power in the highest $m$-mode is expanded in only one $n$-mode, namely a mode having $n=|m|$. However, the integrals $I_{1}$ and $I_{2}$ in Equation (4) can be expanded theoretically in a series of modes with $n=|m| \rightarrow \infty$. As a consequence, the tail of the truncated $M$-spectrum will end up lower than the under-truncated $M$ spectrum. To avoid this loss in power, the $\mathrm{N}$-spectrum is truncated at $N=M+n_{1}$, where the constant $n_{1}$ is chosen in the way that the error between the truncated and the under-truncated M-spectrum is smaller than a certain threshold $B_{F F}(\mathrm{~dB})$. Here again, $F F$ refers to far-field data, and $B$ now denotes a constant threshold that is applied on the $N$-spectrum. In the presented work, the thresholds $A_{F F}$ and $B_{F F}$ were experimentally set to $-130 \mathrm{~dB}$ and $0.5 \mathrm{~dB}$, respectively.

Figure 4 shows the radial component of the reconstructed near-field on the minimum-sphere of the PCB by undertruncating the model $(N=100)$, truncating the model at $\left\lceil k R_{0}\right\rceil$ and at $P_{m\{T E, T M\}}^{n o r m}(\mathrm{~dB})=A_{F F}(\mathrm{~dB})$ together with the fields obtained using a full-wave solver. Remark that the full-wave field contains several large peaks indicating the presence of erroneous high-order modes. Although the reconstructed fields show similar trends, exactly reconstructing the near-field from far-field data is not possible. In contrast, the radial component of the reconstructed near-field of the log-periodic antenna, shown in Figure 5, exhibits almost exact correspondence to the full-wave field. This means that in this case the radiated power is sufficiently large compared to the reactive fields in the near-field, allowing one to reconstruct its near-field from far-field data only with extremely high precision.

\section{B. Near-field data}

In contrast to far-field data, near-field data exhibit a monotonically decreasing radiated power spectrum, as a result of which it is not possible to derive a truncation criterion based on the radiated power spectrum. Therefore, the reactive power spectrum, which seems to asymptotically approach a lower bound, is used to derive a truncation criterion for nearfield models. Section III-B discussed among others how Efields and $\mathrm{H}$-fields lead to discrepancies in their corresponding reactive power spectra from a certain $n$ onwards. In order to see which spectrum is the correct one, Figures $6 \mathrm{a}$ and $6 \mathrm{~b}$ show the radial component of the electric field reconstructed using TM-modes expanded from magnetic fields and the radial component of the magnetic field reconstructed using TE-modes expanded from electric fields. No truncation criterion was applied (under-truncation). One immediately remarks the noisy large-valued graphs which can only be caused by large errors in these TE- and TM-spectra. To obtain a solid truncation criterion based on the reactive power spectrum, near-field models have to be computed from both electric and magnetic tangential fields on the minimum-sphere:

- TE-modes: computed from tangential magnetic fields

- TM-modes: computed from tangential electric fields

Similar to the far-field truncation criterion, only modes for which $\omega W_{m\{T E, T M\}}^{\text {norm }}(\mathrm{dB})>A_{N F}(\mathrm{~dB})$ are kept. Now, $N F$ refers to expanding near-field data, while the threshold $A$ reflects truncating the $M$-spectrum, identically to Section IV-A.

Using both electric and magnetic field data sampled on the minimum-sphere to compute the model and after truncating this model based on the normalized reactive power spectra $\omega W_{m\{T E, T M\}}^{n o r m}$, the reconstructed near-fields of the inverted-F antenna as shown in Figures $6 \mathrm{c}$ and $6 \mathrm{~g}$ are obtained. Note a sufficiently good correspondence between the full-wave fields, shown in Figures $6 \mathrm{~d}$ and $6 \mathrm{~h}$, and the reconstructed fields. The accuracy of the models can easily be decreased or increased by changing the value of $A_{N F}$, which is set experimentally to $-70 \mathrm{~dB}$ in the given examples. In this notation, $A$ reflects truncating the $M$-spectrum and $N F$ refers to near-field data. As a result, each degree $m$ that is being left out from the model, will not contribute more than $-70 \mathrm{~dB}$ compared to the total stored reactive power. The latter is related to the sum of the squared values of the electric and magnetic fields. Analogous to the proposed far-field truncation criterion, the $\mathrm{N}$-spectrum is truncated at $N=M+n_{1}$, where $n_{1}$ is a constant chosen so that the truncated M-spectrum and the under-truncated $\mathrm{M}$-spectrum have a negligible discrepancy, which is captured by the threshold $B_{N F}$. In the latter, the 


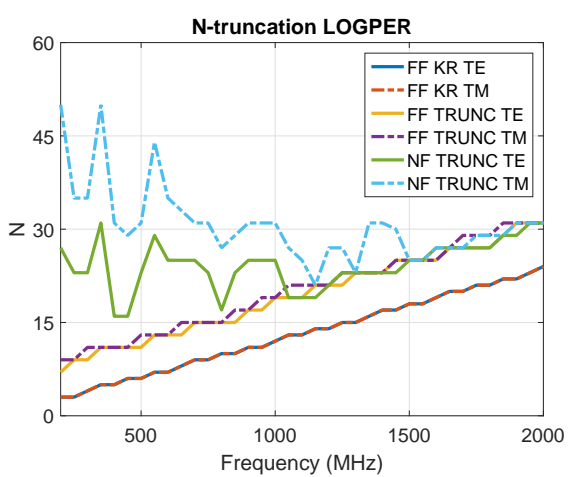

(a)

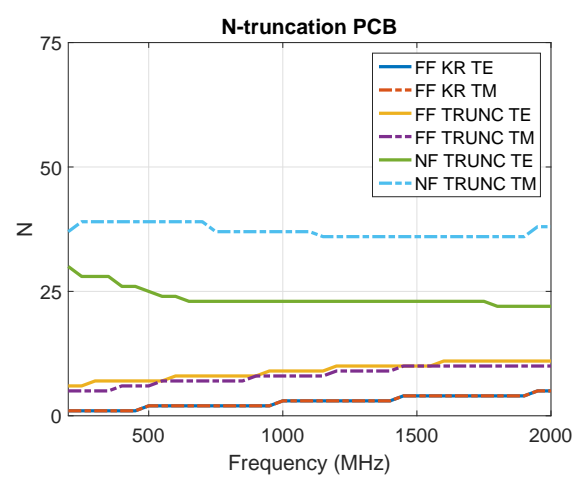

(b)

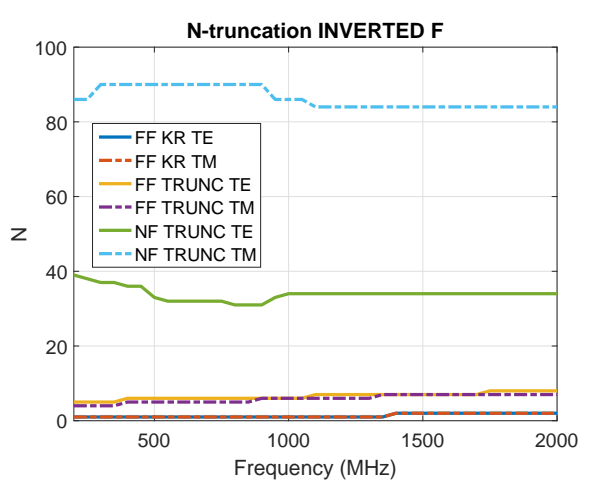

(c)

Fig. 7. TE- and TM- truncation of the log-periodic antenna (a), the PCB (b), and the inverted-F antenna (c) model based on 3 different approaches: (i) FF KR TE/TM: Truncating FF-model (TE/TM) @ $N=\left\lceil k R_{0}\right\rceil$; (ii) FF TRUNC TE/TM: Truncating FF-model (TE/TM) based on its normalized Radiated Power Spectra; and (iii) NF TRUNC TE/TM: Truncating NF-model (TE/TM) based on its normalized Reactive Power Spectra.

notation $B$ now refers to truncating the $N$-spectrum, and this threshold is experimentally set to $3 \mathrm{~dB}$. A lower bound on $N$ is however always set equal to $N=\left\lceil k R_{0}\right\rceil$ where $R_{0}$ is the radius of the minimum-sphere enclosing all radiating sources. Finally, no significant improvement in the reconstructed fields is seen when an under-truncated model $(N=150$, see Figure $6 \mathrm{e})$ is used, while over-truncating the model $(N=20$, see Figure 6f) leads to unacceptably erroneous results. Subsequently, this observation intuitively clarifies the need for an appropriate near-field truncation criterion which performs well for arbitrary DUTs.

\section{Comparison}

All three examples have been modeled and truncated using the proposed techniques described above and the obtained truncations are shown in Figure 7. Notice that the number of additional modes compared to $k R_{0}$ that can be obtained using far-field data is situated between 5 and 10. This value is commonly mentioned in literature as the recommended truncation of spherical wave expansions [17]-[19]. Each of the DUTs seem to act dominantly as TM radiators, which also reveals itself in stronger electric than magnetic fields in the near-field region of the DUT. Remark that the obtained nearfield truncation clearly reveals how large the share of the stored reactive energy is in the near-field region of a DUT, depending on the size and the characteristics of the DUT under study. In this respect, observe for example that the near-field and farfield truncation of the log-periodic antenna nearly coincide between $1.1 \mathrm{GHz}$ and $2 \mathrm{GHz}$, revealing that the contribution of non-radiating or evanescent waves in the antenna's nearfield region is negligible at these frequencies, this in large contrast to e.g. the inverted-F antenna.

\section{CONClusion}

This paper outlined a thorough discussion on spherical wave based macro-models, meant to be applied in system-level EM circuit simulators. Optimal algorithms were discussed that compute fast and efficiently spherical wave spectra of arbitrary DUTs based on tangential near-field or far-field data. Based on a close look at the theory behind SWEs, first-time presented to the authors' knowledge, truncation criteria - both for nearfield and far-field SWE based models - were proposed that ensure convergence of the reconstructed fields in a DUT's near-field region. An optimal algorithm to compute near-field interactions using the above discussed models and the impact of truncation on the computations' accuracy and speed is discussed in [21].

\section{APPENDiX A}

\section{SPHERICAL WAVE FUNCTIONS [17]}

Fields in source-free regions can be expanded in sums of spherical waves [16]:

$$
\begin{aligned}
& \vec{E}(r, \theta, \phi)=\frac{k}{\sqrt{\eta}} \sum_{c=\{1,3\}} \sum_{s=1}^{2} \sum_{n=1}^{\infty} \sum_{m=-n}^{n} Q_{s m n}^{(c)} \times \\
& \vec{F}_{s m n}^{(c)}(r, \theta, \phi) \\
& \vec{H}(r, \theta, \phi)=-i k \sqrt{\eta} \sum_{c=\{1,3\}} \sum_{s=1}^{2} \sum_{n=1}^{\infty} \sum_{m=-n}^{n} Q_{s m n}^{(c)} \times \\
& \vec{F}_{3-s, m, n}^{(c)}(r, \theta, \phi)
\end{aligned}
$$

The spherical wave functions $\vec{F}_{1 m n}^{(c)}(r, \theta, \phi)$ and $\vec{F}_{2, m, n}^{(c)}(r, \theta, \phi)$ are defined as [17]:

$$
\begin{aligned}
& \vec{F}_{1 m n}^{(c)}(r, \theta, \phi)=\frac{1}{\sqrt{2 \pi}} \frac{1}{\sqrt{n(n+1)}}\left(-\frac{m}{|m|}\right)^{m}\left\{R_{1 n}^{(c)}(k r)\right. \\
& \left.\frac{i m \bar{P}_{n}^{|m|}(\cos \theta)}{\sin \theta} e^{i m \phi} \hat{\theta}-R_{1 n}^{(c)}(k r) \frac{d \bar{P}_{n}^{|m|}(\cos \theta)}{d \theta} e^{i m \phi} \hat{\phi}\right\}
\end{aligned}
$$

$$
\begin{aligned}
& \vec{F}_{2 m n}^{(c)}(r, \theta, \phi)=\frac{1}{\sqrt{2 \pi}} \frac{1}{\sqrt{n(n+1)}}\left(-\frac{m}{|m|}\right)^{m}\left\{\frac{n(n+1)}{k r}\right. \\
& R_{1 n}^{(c)}(k r) \bar{P}_{n}^{|m|}(\cos \theta) e^{i m \phi} \hat{r}+R_{2 n}^{(c)}(k r) \frac{d \bar{P}_{n}^{|m|}(\cos \theta)}{d \theta} e^{i m \phi} \hat{\theta} \\
& \left.+R_{2 n}^{(c)}(k r) \frac{i m \bar{P}_{n}^{|m|}(\cos \theta)}{\sin \theta} e^{i m \phi} \hat{\phi}\right\}
\end{aligned}
$$


In these functions, the radial dependence is represented by the function $R_{s n}^{(c)}(k r)$ :

$$
\left\{\begin{array}{l}
R_{1 n}^{(c)}(k r)=z_{n}^{(c)}(k r) \\
R_{2 n}^{(c)}(k r)=\frac{1}{k r} \frac{d}{d(k r)}\left\{k r z_{n}^{(c)}(k r)\right\}
\end{array}\right.
$$

To distinguish between inward and outward traveling waves, the index $c$ is used, following the definition of [29]:

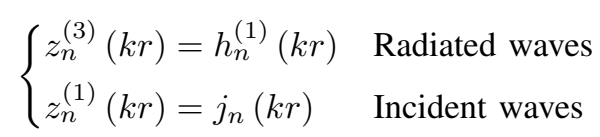

According to [17], the omitted time dependence is assumed to be $e^{-i \omega t}$ and the spherical wave functions are powernormalized such that they each radiate $1 / 2 \mathrm{~W}$.

In the far-field region, the spherical wave functions simplify to their asymptotic forms [17]:

$$
\vec{K}_{s m n}(r, \theta, \phi)=\lim _{k r \rightarrow \infty}\left\{\sqrt{4 \pi} \frac{k r}{e^{i k r}} \vec{F}_{s m n}^{(c)}(r, \theta, \phi)\right\}
$$

The far-field wave functions thus become:

$$
\begin{aligned}
& \vec{K}_{1 m n}(\theta, \phi)=\frac{2}{\sqrt{n(n+1)}}\left(-\frac{m}{|m|}\right)^{m} e^{i m \phi}(-i)^{n+1} \\
& \left\{\frac{i m \bar{P}_{n}^{|m|}(\cos \theta)}{\sin \theta} \hat{\theta}-\frac{d \bar{P}_{n}^{|m|}(\cos \theta)}{d \theta} \hat{\phi}\right\} \\
& \vec{K}_{2 m n}(\theta, \phi)=\frac{2}{\sqrt{n(n+1)}}\left(-\frac{m}{|m|}\right)^{m} e^{i m \phi}(-i)^{n} \\
& \left\{\frac{d \bar{P}_{n}^{|m|}(\cos \theta)}{d \theta} \hat{\theta}+\frac{i m \bar{P}_{n}^{|m|}(\cos \theta)}{\sin \theta} \hat{\phi}\right\}
\end{aligned}
$$

From the orthogonality properties of general spherical wave functions, e.g. given in [17], the orthogonality properties of far-field spherical wave functions can be derived:

$$
\begin{aligned}
& \int_{0}^{2 \pi} \int_{0}^{\pi} \vec{K}_{s m n}(\theta, \phi) \cdot \vec{K}_{\sigma \mu \nu}(\theta, \phi) \sin \theta d \theta d \phi \\
& =4 \pi \delta_{s \sigma} \delta_{m,-\mu} \delta_{n \nu}(-1)^{m}
\end{aligned}
$$

\section{APPENDIX B}

\section{COMPUTATION OF Associated LEGENDRE FUnCTIONS}

This section derives relations allowing one to compute recursively the DST/DCT of $\bar{P}_{n}^{|m|}(\cos \theta), \frac{d \bar{P}_{n}^{|m|}(\cos \theta)}{d \theta}$ and $\frac{m \bar{P}_{n}^{|m|}(\cos \theta)}{\sin \theta}$. Only $n+1$ coefficients need to be computed and stored per function, while under- and overflow problems as well as unstable behaviour of the functions near $0^{\circ}$ and $180^{\circ}$ are now avoided in a straightforward manner.

In contrast to existing algorithms presented in [30], [31], now the recursion is applied directly on the DST/DCT of Associated Legendre Functions (ALFs). Note that:

$$
\bar{P}_{n}^{|m|}(\cos \theta)= \begin{cases}\sum_{k=0}^{n} a_{k} \cos k \theta & \text { if } m=\text { even } \\ \sum_{k=1}^{n} a_{k} \sin k \theta & \text { if } m=\text { odd }\end{cases}
$$

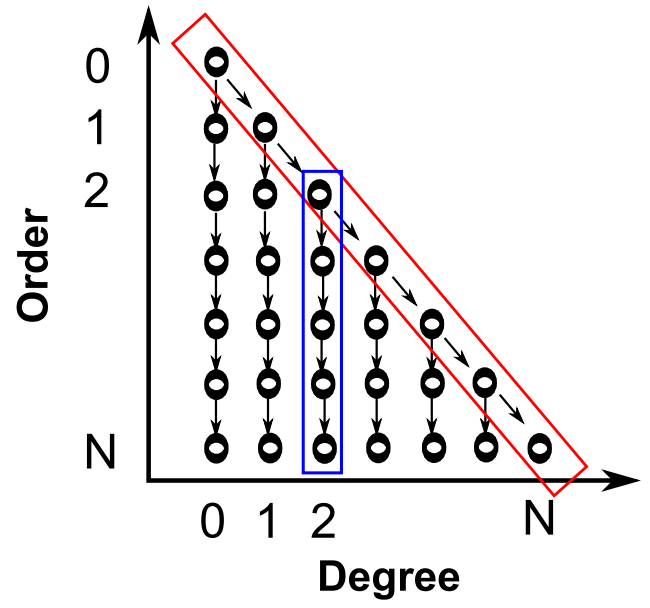

Fig. 8. Schematic recursive computation of Associated Legendre Functions: Standard Forward Column method.

$\frac{\frac{d \bar{P}_{n}^{|m|}(\cos \theta)}{d \theta}}{\frac{m \bar{P}_{n}^{|m|}(\cos \theta)}{\sin \theta}}= \begin{cases}\sum_{k=1}^{n} b_{k} \sin k \theta & \text { if } m=\text { even } \\ \sum_{k=0}^{n} b_{k} \cos k \theta & \text { if } m=\text { odd }\end{cases}$

The DCT/DST of an ALF's derivative is directly evaluated from the ALF's DST/DCT:

$$
\frac{d \bar{P}_{n}^{|m|}(\cos \theta)}{d \theta}= \begin{cases}\sum_{k}-a_{k} k \sin k \theta & \text { if } m=\text { even } \\ \sum_{k}+a_{k} k \cos k \theta & \text { if } m=\text { odd }\end{cases}
$$

Based on the standard forward column recursion [30], the DST/DCT of a set ALFs with maximum degree $N$ is computed recursively as follows (Figure 8):

- Recursively compute the DST/DCT of $\bar{P}_{m}^{|m|}(\cos \theta)$, $\frac{d \bar{P}_{m}^{|m|}(\cos \theta)}{d \theta}$ and $\frac{m \bar{P}_{m}^{|m|}(\cos \theta)}{\sin \theta}$ for $m=0, \ldots, N$

- For each m: perform a forward recursion in $\mathrm{n}$, where $n=\max (1, m), m+1, \ldots, N$

Choose as a starting point $\bar{P}_{0}^{0} \cos \theta=\sqrt{2} / 2$. The first recursion is a one-term recursion in $m$ :

$$
\begin{aligned}
& \frac{m \bar{P}_{m}^{m}(\cos \theta)}{\sin \theta}= \\
& \begin{cases}\sqrt{\frac{m(2 m+1)}{2}} \sin \theta \bar{P}_{m-1}^{m-1}(\cos \theta) & \text { if } m \geq 1 \\
0 & \text { if } m=0\end{cases}
\end{aligned}
$$

The DST/DCT of $\bar{P}_{n}^{|m|}(\cos \theta)$ is obtained by convolving $\frac{m \bar{P}_{m}^{|m|}(\cos \theta)}{\sin \theta}$ and $\sin \theta / m$. Therefore, their DST/DCT is first transformed to the corresponding DFT and afterwards the resulting DFT is back-transformed to the appropriate DST/DCT depending on the ALF's degree $m$. Subsequently, $\frac{d \bar{P}_{m}^{|m|}(\cos \theta)}{d \theta}$ is evaluated using Equation (20).

In the second step, use $\bar{P}_{m}^{m}(\cos \theta)$ as a seed to compute the ALFs for $n=\max (1, m), \ldots, N$. The case for $m=0$ needs to be handled as a special case in order to avoid a division by 0 : 


$$
\begin{aligned}
& \bar{P}_{n}^{0}(\cos \theta)= \\
& \begin{cases}a_{n 0} \cos \theta \bar{P}_{n-1}^{0}(\cos \theta)-b_{n 0} \bar{P}_{n-2}^{0}(\cos \theta) & \text { if } n>1 \\
a_{n 0} \cos \theta \bar{P}_{n-1}^{0}(\cos \theta) & \text { if } n=1\end{cases}
\end{aligned}
$$

where $a_{n 0}$ and $b_{n 0}$ are defined as:

$$
\begin{aligned}
& a_{n 0}=\sqrt{\frac{(2 n-1)(2 n+1)}{n^{2}}} \\
& b_{n 0}=\sqrt{\frac{(2 n+1)(n-1)^{2}}{n^{2}(2 n-3)}}
\end{aligned}
$$

To avoid the factor $\cos \theta$ causing an unstable recusion, for $m \geq 1$ the following recursion is used:

$$
\begin{aligned}
& \frac{m \bar{P}_{n+1}^{m}(\cos \theta)}{\sin \theta}= \\
& \begin{cases}\left\{m \frac{d \bar{P}_{n}^{m}(\cos \theta)}{d \theta}+n F_{(n, m)}^{-}\right. & \text {if } n>m+1 \\
\left.\left(\frac{m \bar{P}_{n-2}^{m}(\cos \theta)}{\sin \theta}\right)\right\} \frac{F_{(n, m)}^{+}}{n-1} & \\
m \frac{d \bar{P}_{n}^{m}(\cos \theta)}{d \theta} \frac{F_{(n, m)}^{+}}{n-1} & \text { if } n=m+1\end{cases}
\end{aligned}
$$

where $F_{(n, m)}^{-}$and $F_{(n, m)}^{+}$are defined as:

$$
\begin{aligned}
& F_{(n, m)}^{-}=\sqrt{\frac{(n-1+m)(n-1-m)}{(2 n-3)(2 n-1)}} \\
& F_{(n, m)}^{+}=\sqrt{\frac{(2 n-1)(2 n+1)}{(n-m)(n+m)}}
\end{aligned}
$$

ALF transforms with a bandwidth of $10,50,100$ and 200 are computed in $0.05 \mathrm{~s}, 0.64 \mathrm{~s}, 2.55 \mathrm{~s}$ and $11.55 \mathrm{~s}$, respectively on $2.1 \mathrm{GHz}$ dual core processor.

\section{REFERENCES}

[1] CST Design Studio. Computer Simulation Technology. [Online]. Available: https://www.cst.com/Products/CSTDS

[2] Keysight ADS. Keysight Technologies. [Online]. Available: http: //www.keysight.com/en/pc-1297113/advanced-design-system-ads

[3] Mentor Graphics System Modeling. Mentor Graphics. [Online]. Available: http://www.mentor.com/products/sm/

[4] Cadence Sigrity. Cadence. [Online]. Available: http://www.cadence. com/products/sigrity/pages/default.aspx

[5] EMCoS EMC Studio. EMCoS. [Online]. Available: http://www.emcos. com/?products=emc-studio

[6] X. Tong and D. Thomas, "Modeling electromagnetic emissions from printed circuit boards in closed environments using equivalent dipoles," IEEE Trans. Electromagn. Compat., vol. 52, no. 2, pp. 462-470, May 2010. [Online]. Available: http://ieeexplore.ieee.org/xpls/abs $\backslash$ all.jsp? arnumber $=5437259$

[7] Y. Gilabert, "Modélisation des Émissions Rayonnées de Composants Électroniques," Ph.D. dissertation, École Doct. Sci. Phys. Math. Inform. Ing. (SPMII), Univ. Rouen, 2007.

[8] B. V. Thielen, "Method for the calculation of mutual coupling between discontinuities in planar circuits," IEEE Trans. Microw. Theory Tech., vol. 50, no. 1, pp. 155-164, Jan. 2002. [Online]. Available: http://ieeexplore.ieee.org/xpls/abs $\backslash$ all.jsp?arnumber=981260

[9] F. de Adana and M. Cátedra, "An Effective Technique for System-Level Prediction of the Radiated Emissions of Unknown Sources Inside LowCavities Using Unit-Level Measurements," IEEE Trans. Electromagn. Compat., vol. 51, no. 2, pp. 181-191, May 2009. [Online]. Available: http://ieeexplore.ieee.org/xpls/abs $\backslash \_a l l . j s p ? a r n u m b e r=4939360$
[10] I. Erdin and M. Nakhla, "Circuit analysis of electromagnetic radiation and field coupling effects for networks with embedded full-wave modules," IEEE Trans. Electromagn. Compat., vol. 42, no. 4, pp. 449-460, Nov. 2000. [Online]. Available: http://ieeexplore.iee.org/xpls/ abs $\backslash$ all.jsp?arnumber $=902314$

[11] J. Balbastre and L. Nuo, "Assessment of complex radiated EMC problems involving slotted enclosures using a 2-D generalized circuital approach," IEEE Trans. Electromagn. Compat., vol. 49, no. 3, pp. 543-552, Aug. 2007. [Online]. Available: http://ieeexplore.iee.org/ $x p l s / a b s \backslash$ all.jsp?arnumber $=4295210$

[12] D. Drogoudis, J. V. Hese, B. Boesman, and D. Pissoort, "Combined Circuit / Full-Wave Simulations for ElectroMagnetic Immunity Studies Based on an Extended S-Parameter Formulation," in 2014 IEEE 18th Workshop Sign. Power Integr. (SPI), 2014, pp. 4-7.

[13] T. B. Hansen, "Exact plane-wave expansion with directional spectrum: Application to transmitting and receiving Antennas," IEEE Trans. Antennas Propag., vol. 62, no. 8, pp. 4187-4198, Aug. 2014.

[14] F. J. Demuynck, G. A. E. Vandenbosch, and A. R. V. D. Capelle, "The Expansion Wave Concept Part I : Efficient Calculation of Spatial Green s Functions in a Stratified Dielectric Medium," IEEE Trans. Antennas Propag., vol. 46, no. 3, pp. 397-406, Mar. 1998.

[15] G. A. E. Vandenbosch and F. J. Demuynck, "The expansion wave concept-Part II: A new way to model mutual coupling in microstrip arrays," IEEE Trans. Antennas Propag., vol. 46, no. 3, pp. 407-413, Mar. 1998.

[16] J. A. Stratton, Electromagnetic theory, 1st ed. McGraw-Hill New York, 1941, ch. VII Spherical Waves, pp. 392-423.

[17] J. E. Hansen, Ed., Spherical Near-field Antenna Measurements, ser. Electromagnetic Waves. IET, 1988, vol. 26. [Online]. Available: http://digital-library.theiet.org/content/books/ew/pbew026e

[18] P. Kralicek, "Makromodellierung des strahlungsverhaltens von subsystemen zur systemanalyse," Ph.D. dissertation, Fakult. Elektrotechn. Inform., Univ. Hannover, 2006.

[19] M. Haynes and M. Moghaddam, "Multipole and s-parameter antenna and propagation model," IEEE Trans. Antennas Propag., vol. 59, no. 1, pp. 225-235, Jan. 2011.

[20] F. Jensen and A. Frandsen, "On the number of modes in spherical wave expansions," in Proc. 26th AMTA, 2004, pp. 489-494. [Online]. Available: https://www.ticra.com/sites/default/files/ Numberofsphericalmodes.pdf

[21] B. Boesman, G. Gielen, G. A. E. Vandenbosch, and D. Pissoort, "Spherical Wave Based Macro-Models for Efficient System-Level EMC Analysis in Circuit Simulators - Part II: Optimized Calculations of DUTDUT Interactions," IEEE Trans. Electromagn. Compat., to be published.

[22] F. Kervel, Design Note DN023: $868 \mathrm{MHz}, 915 \mathrm{MHz}$ and $955 \mathrm{MHz}$ Inverted F Antenna, Texas Instruments, 2011. [Online]. Available: http://www.ti.com/lit/an/swra228c/swra228c.pdf

[23] Keysight Empro (2015.01). Keysight Technologies. [Online]. Available: http://www.home.agilent.com/en/pc-1297143/ empro-3d-em-simulation-software

[24] R. Pirkl, "Spherical wave scattering matrix description of antenna coupling in arbitrary environments," IEEE Trans. Antennas Propag., vol. 60, no. 12, pp. 5654-5662, Dec. 2012.

[25] B. Boesman, D. Pissoort, G. Gielen, and G. A. E. Vandenbosch, "A circuit approach to compute near-field interactions based on an efficient implementation of the spherical wave expansion," in 2014 Int. Symp. Electromagn. Compat., 2014, pp. 91-96. [Online]. Available: http: //ieeexplore.ieee.org/lpdocs/epic03/wrapper.htm?arnumber=6930883

[26] A. Voors. 4nec2. [Online]. Available: http://www.qsl.net/4nec2/

[27] T. V. Hansen, O. S. Kim, and O. Breinbjerg, "Stored Energy and Quality Factor of Spherical Wave Functionsin Relation to Spherical Antennas With Material Cores," IEEE Trans. Antennas Propag., vol. 60, no. 3, pp. 1281-1290, Mar. 2012. [Online]. Available: http: //ieeexplore.ieee.org/lpdocs/epic03/wrapper.htm?arnumber $=6107533$

[28] R. Collin and S. Rothschild, "Evaluation of antenna Q," IEEE Trans. Antennas Propag., vol. 12, no. 1, pp. 23-27, Jan. 1964 [Online]. Available: http://ieeexplore.ieee.org/lpdocs/epic03/wrapper. htm?arnumber $=1138151$

[29] A. Yaghjian, "Scattering-matrix analysis of linear periodic arrays," IEEE Trans. Antennas Propag., vol. 50, no. 8, pp. 1050-1064, Aug. 2002.

[30] S. A. Holmes and W. E. Featherstone, "A unified approach to the Clenshaw summation and the recursive computation of very high degree and order normalised associated Legendre functions," J. Geodesy, vol. 76, no. 5, pp. 279-299, May 2002. [Online]. Available: http://www.springerlink.com/openurl.asp?genre=article $\backslash \& i d=$ doi:10.1007/s00190-002-0216-2 
[31] S. Claessens, "New relations among associated Legendre functions and spherical harmonics," J. Geodesy, vol. 79, no. 6-7, pp. 398-406, Aug. 2005. [Online]. Available: http://www.springerlink.com/index/10.1007/ s00190-005-0483-9

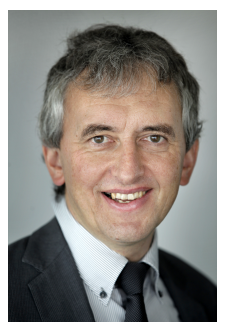

Georges G.E. GIELEN received the MSc and $\mathrm{PhD}$ degrees in Electrical Engineering from the Katholieke Universiteit Leuven, Belgium, in 1986 and 1990, respectively. In 1990, he was appointed as a postdoctoral research assistant and visiting lecturer at the Department of Electrical Engineering and Computer Science of the University of California, Berkeley. From 1991 to 1993, he was a postdoctoral research assistant of the Belgian National Fund of Scientific Research at the ESAT laboratory of the Katholieke Universiteit Leuven. In 1993, he was appointed assistant professor at the Katholieke Universiteit Leuven, where he later promoted to associate professor and finally full professor in 2000. From 2007 till 2012 he was the Head of the Microelectronics and Sensors (MICAS) research division, which included five professors and more than $70 \mathrm{PhD}$ students. In August 2012 he became the Chair of the Department of Electrical Engineering (ESAT) at the Katholieke Universiteit Leuven. He is also the Chair of the Leuven ICT (LICT) research center, and the PI coordinator of the Leuven Center of Excellence called CHIPS. In August 2013 he was appointed vice-rector of KU Leuven responsible for the group Science, Engineering \& Technology, which he executes till today.

His research interests are in the design of analog and mixed-signal integrated circuits, and especially in analog and mixed-signal CAD tools and design automation (modeling, simulation and symbolic analysis, analog synthesis, analog layout generation, analog and mixed-signal testing). $\mathrm{He}$ is coordinator or partner of several (industrial) research projects in this area, including several European projects (EU, MEDEA/CATRENE, ESA). He has authored or coauthored 7 books and more than 450 papers in edited books, international journals and conference proceedings. He regularly is a member of the Program Committees of international conferences (DAC, ICCAD, ISCAS, DATE, CICC...), and served as General Chair of the DATE conference in 2006 and of the ICCAD conference in 2007. He is currently the Chair of EDAA. He serves regularly as member of editorial boards of international journals (IEEE Transactions on Circuits and Systems, IEEE Transactions on ComputerAided Design, Springer International Journal on Analog Integrated Circuits and Signal Processing, Elsevier Integration).

He received the 1995 Best Paper Award in the John Wiley international journal on Circuit Theory and Applications, and was the 1997 Laureate of the Belgian Royal Academy on Sciences, Literature and Arts in the discipline of Engineering. He received the 2000 Alcatel Award from the Belgian National Fund of Scientific Research for his innovative research in telecommunications, and won the DATE 2004 conference Best Paper Award. He served as elected member of the Board of Governors of the IEEE Circuits And Systems (CAS) society, as appointed member of the Board of Governors of the IEEE Council on Electronic Design Automation (CEDA), and as Chairman of the IEEE Benelux CAS Chapter. He served as the President of the IEEE Circuits And Systems (CAS) Society in 2005, and as the Chair of the IEEE Benelux Section in 2009-2010. He was elected DATE Fellow in 2007, and received the IEEE Computer Society Outstanding Contribution Award and the IEEE Circuits and Systems Society Meritorious Service Award in 2007. He received the IEEE Council on Electronic Design Automation recognition award in 2014, and the IEEE Circuits and Systems Mac Van Valkenburg career award in 2015. He is Fellow of the IEEE since 2002.

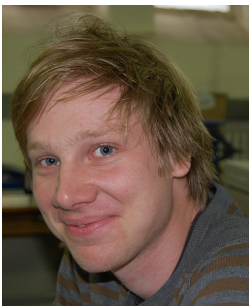

Bart BOESMAN was born in 1986. He received the M.S. degree in electrical engineering from the University College Katholieke Hogeschool Sint-Lieven, Ghent, Belgium in 2009. Since 2010, he is working as a research assistant at the ReMI research lab of $\mathrm{KU}$ Leuven at the KU Leuven technology campus Ostend. In 2011, he started working towards the $\mathrm{PhD}$ degree in electrical engineering, focussing on computational electromagnetics and simulation tools for efficient system-level EMC analysis. His further research interests include high-speed PCB design, signal and power integrity, cable radiation and antenna analysis. 


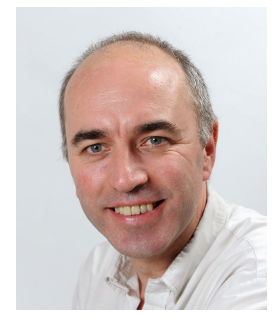

Guy A. E. VANDENBOSCH received the M.S. and $\mathrm{Ph} . \mathrm{D}$. degrees in Electrical Engineering from the Katholieke Universiteit Leuven, Leuven, Belgium, in 1985 and 1991, respectively. Since 1993, he has been a Lecturer, and since 2005, a Full Professor at the same university. Guy Vandenbosch has taught or teaches courses on Electromagnetic Waves, Antennas, Electromagnetic Compatibility, Electrical Engineering, Electronics, and Electrical Energy, and Digital Steer- and Measuring Techniques in Physics. His research interests are in the area of electromagnetic theory, computational electromagnetics, planar antennas and circuits, nano-electromagnetics, EM radiation, EMC, and bio-electromagnetics. His work has been published in ca. 225 papers in international journals and has lead to ca. 320 presentations at international conferences. From 2001 to 2007, he was the President of SITEL, the Belgian Society of Engineers in Telecommunication and Electronics. From 2008 - 2014, he was a member of the board of FITCE Belgium, the Belgian branch of the Federation of Telecommunications Engineers of the European Union. In the period 19992004, he was vice-chairman, and in the period 2005-2009 secretary of the IEEE Benelux Chapter on Antennas en Propagation. Currently he holds the position of chairman of this Chapter. In the period 2002-2004 he was secretary of the IEEE Benelux Chapter on EMC. In the period 2012-2014 he was secretary of the Belgian National Committee for Radio-electricity (URSI), where he is also in charge of commission E. From September to December 2014, he was a visiting professor at Tsinghua University, Beijing, China.

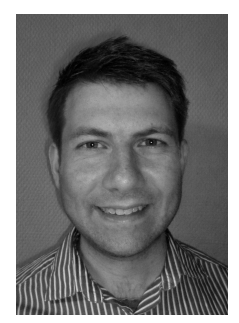

Davy PISSOORT (M09, SM13) was born in 1978. He received the M.S. and Ph.D. degrees in electrical engineering from Ghent University, Ghent, Belgium, in 2001 and 2005, respectively. From October 2005 till October 2006, he was a Postdoctoral Researcher at Ghent University, Belgium. From November 2006 till July 2009, he was a Research Engineer at the Eesof-EDA Department of Agilent Technologies in Belgium. Since August 2009, he is Assistant Professor at KU Leuven, Technology Campus Ostend, Belgium, where he is also head of the research group Reliability in Mechatronics and ICT. His current research interests include the development of fast and efficint electromagnetic modelling methods for EMC, SI and PI, the development of characterization methods for shielding materials and gaskets, EMI Risk Management as well as the analysis and testing of the mechanical and thermal reliability of electronic modules. He is Senior Member of the IEEE EMC Society and member of the International Steering Committee of EMC Europe. In September 2013 he was vice-chair for the EMC Europe 2013 Symposium in Brugge, Belgium. In August 2015 he was co-chair for the Workshops and Tutorials at the joined IEEE EMC and EMC Europe symposium in Dresden, Germany. 